\title{
An Application of Mobile Cloud Computing in the GPS of Taxi
}

\author{
Wen-qing Zhao1, a, Yu-xin Dong2, b , Jian-jun Chen3,c and Yao \\ Li4,d \\ 1-4Institute of control and computer engineering, North China Electric \\ Power University, Baoding, 071003, Hebei, China \\ ajbzwq@126.com,b448221045@qq.com,c1195998781@qq.com, \\ d1308611677@qq.com
}

\begin{abstract}
This paper is designed for the filtering algorithms of taxi GPS data, as well as vehicle trajectory statistics and prediction algorithm which based on the theory of spatial-temporal data mining algorithm. On the basis of these algorithms, we developed intelligent taxi forecasting system based on mobile cloud computing. Through advanced intelligent algorithms and useful data of vehicle GPS which were got by cloud-based platform and service, we can determine the densest area of the empty taxi where is closest to the user and show it on a mobile device. Thus it can provide a reference for the user.

Keywords: Mobile cloud computing; Spatio-temporal data mining; Prediction of vehicle trajectory.

\section{Introduction}

In recent years, along with urban development, the issues of taxi difficulty have become increasingly prominent, and the existing taxi software has the following problems. First of all, we cannot take a taxi without software; second, drivers pick passengers; third, traffic safety hazard. Today, the taxi of major cities are loaded with GPS chips which would periodically transmit its location information to the service center. Thereby, they together formed a large-scale mobile taxi trajectory data. In this paper, in order to enable users to easily and quickly find the empty taxi as well as reduce waiting time while not causing the above disadvantages, we designed a taxi forecasting system of android based on mobile cloud computing platform, to provide users with efficient and practical travel references.
\end{abstract}

System Architecture. System architecture is mainly divided into three parts: One is the mobile client, the second is the cloud server, the last is secure communication between the client and service side of the cloud (as shown in Fig. $1)$. 


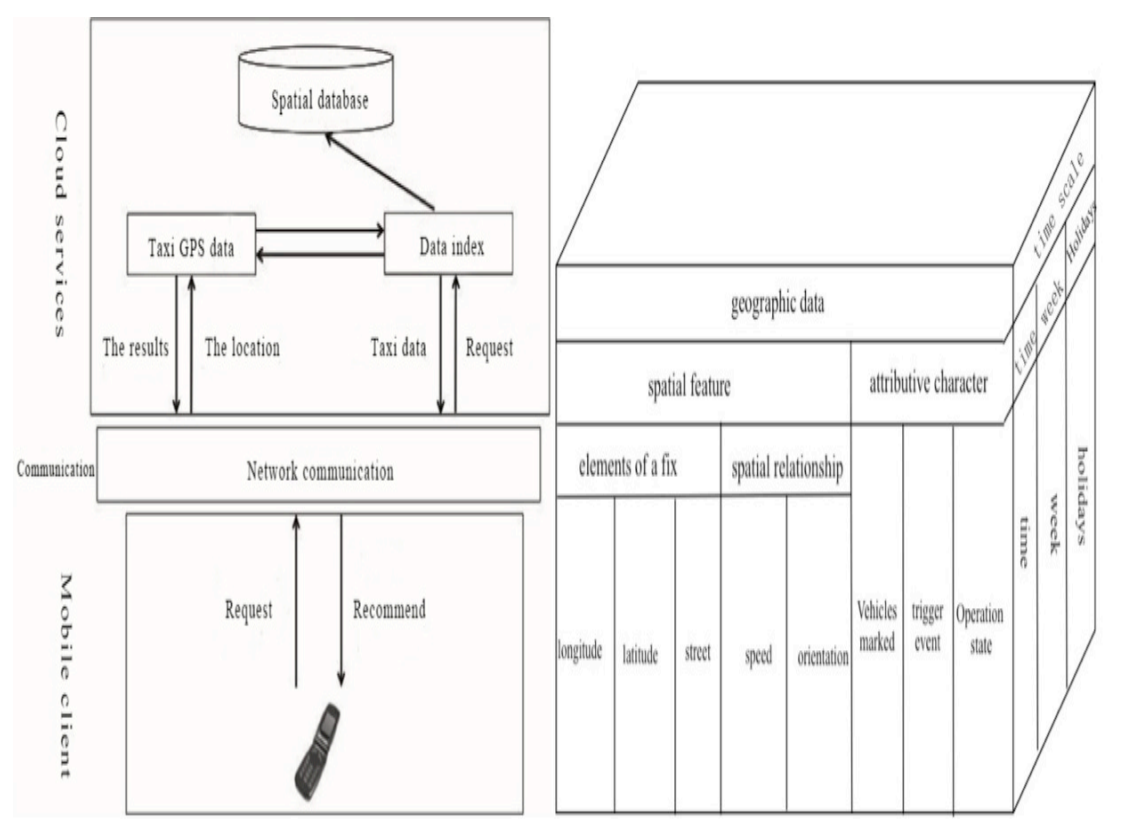

Fig.1 Taxi forecasting system and the multidimensional data structure diagram of GPS

The Data Mining of Taxi GPS. According to the data at our disposal, we build a taxi forecasting system of multidimensional spatio-temporal data structure diagram as shown in Figure 4. The data mining of Vehicle GPS consists of five modules that are respectively the workflow module, data loading module, data preprocessing module, data mining algorithm module and results display module. The workflow module is for vehicle GPS data mining procedures, total control of modules and scheduling functions; data loading module loads the data of user and taxi to storage system in cloud computing platform; the required data for mining is preprocessed by the parallel ETL module; data mining algorithm module can provide GPS data to the cloud computing platform and achieve the tasks of vehicle trajectory prediction algorithm performed by the cloud platform. They feedback the results and are stored in the storage system. Result display module returns the calculation result to the user.

\section{The Match between GPS Data and Road Network.}

(1) Screening feasible sections. Converted to rover coordinates for the center and fixed a circular area on the map, the default value of screening range $\mathrm{R}$ is the radius. We record all sections within the region as alternative road as well (as shown in Fig.2). 


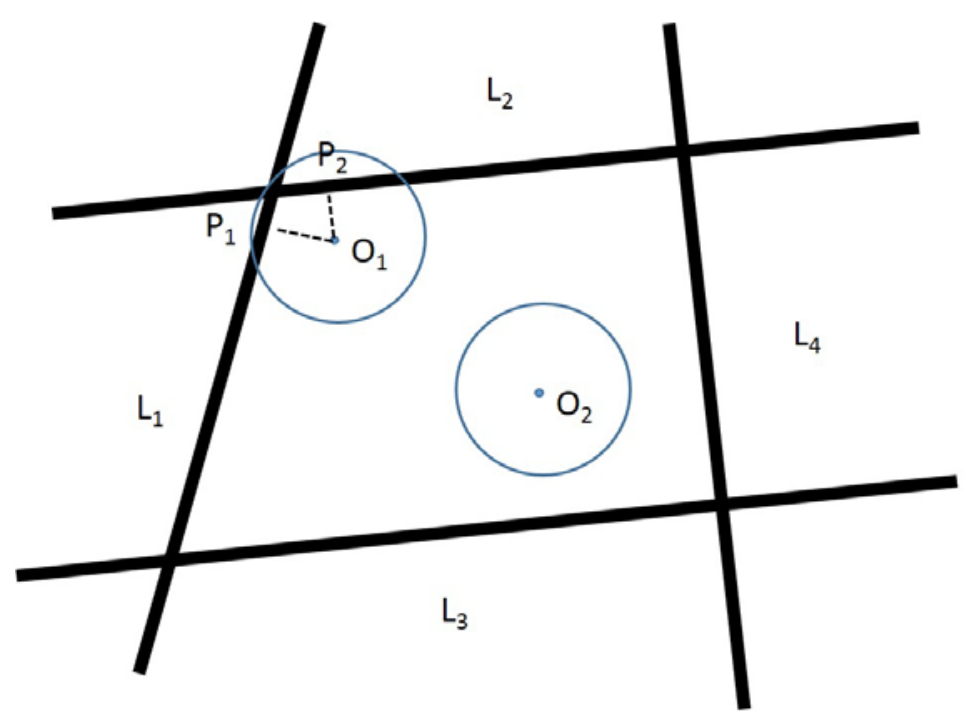

Fig.2 Matching GPS vehicle trajectory with road network.

As for Map data for all the line segments in the section of data within a map, we calculate the rovers coordinates to the line $(\mathrm{x}, \mathrm{y})$ to the lines $\mathrm{i}(\mathrm{i}=1,2,3 \ldots \mathrm{n})$ projection distance [1].

$$
\mathrm{d}_{\mathrm{i}}^{2}=\frac{\left[\left(y_{\mathrm{i} 2}-\mathrm{y}_{\mathrm{i} 1}\right) \times \mathrm{x}-\left(\mathrm{x}_{\mathrm{i} 2}-\mathrm{x}_{\mathrm{i} 1}\right) \times \mathrm{y}^{+} \mathrm{x}_{\mathrm{i} 2} \times \mathrm{y}_{\mathrm{i} 1}-\mathrm{x}_{\mathrm{i} 1} \times \mathrm{y}_{\mathrm{i} 2}\right]^{2}}{\left(\mathrm{x}_{\mathrm{i} 2}{ }^{2}-\mathrm{x}_{\mathrm{i} 1}{ }^{2}\right)^{2}+\left(\mathrm{y}_{\mathrm{i} 2}{ }^{2}-\mathrm{y}_{\mathrm{i} 1}{ }^{2}\right)^{2}}
$$

(1)

Among them, $\left(\mathrm{x}_{\mathrm{i} 1}, \mathrm{y}_{\mathrm{i} 1}\right)$ and $\left(\mathrm{x}_{\mathrm{i} 2}, \mathrm{y}_{\mathrm{i} 2}\right)$ are the end coordinates of corresponding line segment respectively.

(2) Calculate fixed coordinates. Select sections that contains the matching line as the actual section of the Rover, and calculates the Rovers at the projection point coordinates on the fold line. As amended, coordinates of the Rover survived. Fix coordinates calculation formula [2]. 


$$
\left\{\begin{array}{l}
\mathrm{k}_{\mathrm{i}}=\frac{y_{\mathrm{i} 2}-\mathrm{y}_{\mathrm{i} 1}}{\mathrm{x}_{\mathrm{i} 2}-\mathrm{x}_{\mathrm{i} 1}} \\
\mathrm{x}_{\mathrm{p}}=\frac{\mathrm{x}+\mathrm{k}_{\mathrm{i}} \times\left(y+\mathrm{y}_{\mathrm{i} 1}\right)+\mathrm{k}_{\mathrm{i}}^{2} \times \mathrm{x}_{\mathrm{i} 1}}{1+\mathrm{k}_{\mathrm{i}}^{2}} \\
\mathrm{y}_{\mathrm{p}}=\mathrm{k}_{\mathrm{i}} \times\left(\mathrm{x}_{\mathrm{p}}-\mathrm{x}_{\mathrm{i} 1}\right)+\mathrm{y}_{\mathrm{i} 1}
\end{array}\right.
$$

(2)

Where (x, y) coordinates correction for vehicle.

(3) Distinguish between upper and lower sections. Based on probe vehicle data in the front toward data, as well as according to the angle between the road network sections up and down and due north direction, the section on the vehicles were matched to the angle between the directions of the smaller. Therefore distinguishing between vehicles driving directions.

Results matching the entire map is as shown in Fig.3, with red dots representing the data after conversion to urban construction coordinates, and match point to sections of green data as well.

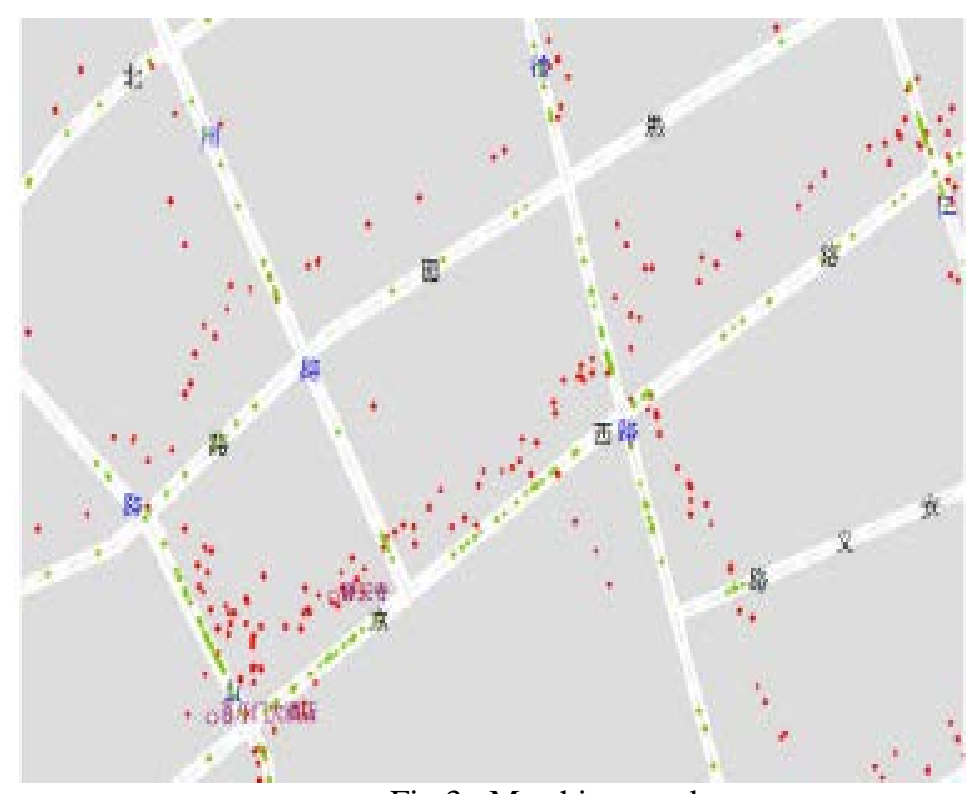

Fig.3 Matching results 
Filter GPS Data through the Cloud. In the taxi system of mobile cloud computing, mobile user data which are submitted to the server from client consist of two parts: the user's current location (latitude and longitude position coordinates of a point) and current time. The server will certainly process the result feedback to the user based on historical data probability of hail a cab around here.

Here we use the Dijkstra algorithm. The main features are an extension to the starting point for the center to the outer layer, until extending to the end. Dijkstra shortest path algorithm can arrive at the optimal solution, because of its calculation of the node traversal, inefficiently (the time complexity is $\mathrm{O}\left(\mathrm{n}^{3}\right)$, the space complexity is $\mathrm{O}\left(\mathrm{n}^{2}\right)$ ). However, unless the root net Change, the calculation process takes just one time. In practice Dijkstra is acceptable.

The calculation process can have a natural parallelism. System use distributed and parallel computing to speed up the calculation of the offline process. To be specific, having Eigen wert R and rood net $\mathrm{G}$ with the given size of $\mathrm{n}$, and setting system usage $\mathrm{P} \leq$ processors $n$, every processor calculates " $\mathrm{n} / \mathrm{p}$ " a starting point to the right to take the shortest path between all points at most and each processor has a copy of the G. In practice, system uses parallel algorithms in formula [2] to figure out the shortest path point to point. And it parallels Floyd-Warshall by matrix multiplication algorithm and its time complexity is $O\left(" \mathrm{n} / \mathrm{p} " \mathrm{n}^{2}\right)$, because the calculation process does not require communication between processors. In theory, the parallel optimization realizes linear speedup (value is $\mathrm{P}$ ).

Vehicle Trajectory Prediction. Track-intensive regions for a taxi passenger demand hotspots, and taxi passengers have more data to be recorded. In order to allow mobile users to quickly get featured in a cab, mainly use offline mode, the cloud collects the taxi GPS tracking data of previous years and we store the data mining results into the database. When users make a request to the cloud, the result from the database is returned to the client directly. The benefits of such an approach are that it can handle massive data, have high precision, consume time without limit, and refresh regularly, but it has higher requirements for reasonableness of the regional division.

For fewer passengers and more remote areas, taxis have less tracking data. And with a lot of randomness, they can use real-time feedback. After a user sends a request, cloud server received and presented the request to the Hadoop cluster. Being calculated by the cluster, cluster send the result to the server, and then return to the user. Due to the small amount of data, processing speed is within an acceptable range.

In order to reduce the computational cost of online search and achieve real-time search, offline storage system precalculated all points with shortest-path problem, and used an inversion table to store the calculated results.

Client-Side Design. Using powerful cloud server for data processing and then displaying result on the phone, it not only solves the burden of mobile data processing, but also brings a lot of convenience to data updating. This client has 3 core modules. The first is location. The second is to send location data, namely sent the latitude and longitude information of passenger location to the server. 
And the third is the electronic map display. Display density recommend idle taxis running position on an electronic map of a mobile device.

Experiment. By the cloud data processing, we got the taxi recommendation of six places nearby North China Electric Power University in workdays and off days. From 10:00 to 13:00, the mobile phone showed six recommended places to take a taxi, as shown in the Fig. 4 below. We recorded the numbers of taxi by artificial statistics in 10 days, then we found that it is accordant to the recommended result. The accuracy of prediction system is verified.

\begin{tabular}{|l|l|l|l|l|l|l|}
\hline & 3 & 2 & 6 & 1 & 5 & 4 \\
\hline workday & & & & & & \\
\hline
\end{tabular}

\section{口7--10H $\square$ 10--13H $\square$ 13--17H $\square$ 17--20H $\square 20--23 \mathrm{H} \square 23--07 \mathrm{H}$}

Fig.4 Analysis of the passed Qty

\section{References}

[1] Wei C: The computing method of traffic state Research (2003)

[2] Han Y, Pan V, Reif J Efficient parallel algorithms for computing all pair shortest paths in directed graphs Algorithmica, 1997, 17(4) 399-415.

[3] Liao L, Patterson D J, Fox D, et al. Building Personal Maps from GPS Data[C]. In: Proceedings of In Proceedings of International Joint Conference on Artificial Intelligence Workshop "Modeling Others From Observations " , 2005, 249-265.

[4] Pérez-Torres R, Torres-Huitzil C. A power-aware middleware for location \& context aware mobile apps with cloud computing interaction[C]//nformation and Communication Technologies (WICT), 2012 World Congress on. IEEE, 2012: 691-696. 
[5] Tan C, Xie X Z, Deng R Y: Energy-saving algorithm for mobile cloud computing services image positioning system (2013)

[6] Chen S, Lin M, Zhang H. Research of mobile learning system based on cloud computing[C]//e-Education, Entertainment and e-Management (ICEEE), 2011 International Conference on. IEEE, 2011: 121-123. 